\title{
PROPERTIES OF A QSO SAMPLE SELECTED THROUGH VARIABILITY
}

\author{
D. TREVESE \\ Istituto Astronomico, Universita di Roma "La Sapienza" \\ Via G. M. Lancisi, 29 \\ 000161, Roma, Italy \\ D. C. KOO \\ Lick Observatory, Board of Studies in Astronomy and Astrophysics \\ University of California, Santa Cruz, CA 95064 U.S.A. \\ R. G. KRON \\ Yerkes Observatory, University of Chicago \\ Williams Bay, WI 53191 U.S.A.
}

\section{Introduction}

Variability of QSOs serves not only as an independent search technique but also as another parameter to correlate with such properties as luminosity (in radio, optical, Xray), redshift, spectral features, color, etc., so as to better understand AGNs. We present some preliminary results on a variability study of an essentially complete sample using $4 \mathrm{~m}$ prime focus plates of a $0.3 \mathrm{deg}^{2}$ field at the North Galactic Pole (SA57). The faintness of our survey, $\mathrm{B} \approx 23$; the precision of the measurements $(\sigma \leq 0.05 \mathrm{mag})$; and the availability of redshifts for many of the candidates provide an opportunity to study QSO variability in regimes previously unexplored (c.f. Bonoli et al. 1979 or Hawkins 1986).

\section{Sample and Analysis}

Nine $4 \mathrm{~m}$ blue $(\mathrm{J})$ plates in SA 57 spanning 11 years and seven epochs were digitized and reduced to a catalog of magnitudes, with one of the best plates serving as the fiducial one for magnitudes. Only stellar-like objects brighter than $J=22.5$ were selected for further analysis, consisting of computing for each object a weighted rms standard deviation in magnitude $(\sigma)$, after rejecting at most one measurement larger than the mean by 0.67 mag and with weights that depended upon the quality of the plates. More details can be found in Trevese and Kron (1988). Photographic UBVI colors were derived from another SA57 catalog of stellar-like objects used in selecting the QSO candidates of Koo, Kron, and Cudworth (KKC 1986); spectroscopic redshifts are from Koo and Kron (1988). 


\section{Results}

We conclude:

- Variability at our precision and timescales is an excellent independent technique for selection of quasars, although we have so far not confirmed the reality of those candidates not already selected by KKC. For example, to $\mathrm{J}=21.5$, only one KKC QSO among sixteen would be missed by variability defined as $\sigma \geq 0.9$ mag. In other words, nearly $100 \%$ of this complete optically selected QSO sample display variability.

- The sample of $\mathrm{KKC}$ is relatively complete to $\mathrm{J}=21.5$, since only four variable candidates (some of which may be genuine Galactic stars) were not selected by KKC. To a limit fainter by a magnitude, the relative number of variables not detected by $\mathrm{KKC}$ is at most $50 \%$, thus confirming that not a large number of faint QSOs have been missed by the two color technique. These could, of course, be at high redshifts and thus affect the derived evolution of the QSO luminosity function. Spectroscopic confirmation of the variables not in KKC would be an important next step in this project.

- No correlations in variability amplitude and either redshift or absolute luminosity has been detected in our complete, but very small, sample of 27 optically selected QSOs with spectroscopic redshifts. These results can be compared to similar studies of brighter complete samples by Bonoli et al. (1979), who found that nearly all of their sample of 148 optically selected QSOs varied over a four year period with no correlation between the amplitude and luminosity for a subsample of 23 objects; Uomoto et al. (1976), who found a luminosity anticorrelation for a sample of radio loud QSOs; or Netzer and Sheffer (1983), who found no significant redshift correlations.

We acknowledge the support by Kitt Peak National Observatory and its staff for more than a decade that has made this project possible.

\section{References}

Bonoli, F., Braccesi, A., Federici, L., Zitelli, V., and Formiggini, L. 1979, A. A. Suppl., 35, 391.

Hawkins, M. R. S. 1986, M.N.R.A.S., 219, 417.

Koo, D. C., Kron, R. G., and Cudworth, K.M. 1986, Pub. A.S.P.,98, 285 (KKC).

Koo, D. C. and Kron, R. G. 1988, Ap. J., 325, 92.

Netzer, H. and Sheffer, Y. 1983, M.N.R.A.S., 203, 935.

Trevese, D. and Kron, R. G. 1988, Pub. A.S.P., in press.

Uomoto, A. K., Wills, B. J., Wills, D. 1976, A. J.,81, 905. 